\title{
ASPECTOS CONVERGENTES DEL PENSAMIENTO CRÍTICO Y LAS CUESTIONES SOCIOCIENTÍFICAS*
}

\section{Convergence aspects of the critical thought and socio-scientific issues}

\author{
Recibido: 22 de agosto de 2013 • Aprobado: 14 de abril de 2014 \\ Nidia Yaneth Torres Merchán* * \\ Jordi Solbes Matarredona ***
}

\begin{abstract}
Resumen
En este trabajo se presenta un análisis de la conceptualización del pensamiento crítico, a partir de referentes de la filosofía y de la didáctica de las ciencias, en un grupo de estudiantes del ámbito universitario. Se observa que las interpretaciones realizadas por los participantes, con respecto a este tipo de pensamiento, articulan aspectos importantes de las cuestiones socio científicas (CSC) relacionadas con el cuestionamiento de la información, la multidimensionalidad y la toma de decisiones.
\end{abstract}

Palabras clave: pensamiento crítico, cuestiones socio-científicas, enseñanza de las ciencias.

\begin{abstract}
In this work, we present an analysis of the conceptualization of the critical thought, based on references from the Philosophy and Didactics of science, in a group of university students. We found that interpretations made by the participants in front of this kind of thought involve important aspects of socioscientific questions (SCQ), related to the questioning of information, multidimensionality of science and decision making.
\end{abstract}

Keywords: critical thought, socio-scientific questions, science education issues.

* Este trabajo presenta resultados parciales de la tesis doctoral sobre Pensamiento Crítico y Cuestiones Sociocientíficas en la Universidad de Valencia España.

** Profesora de la Universidad Pedagógica y Tecnológica de Tunja. Correo electrónico: nidia.torres@uptc.edu.co

*** Doctor en Ciencias Físicas. Profesor Titular de Didáctica de las Ciencias Experimentales. Universidad de Valencia. Correo electrónico: jordi.solbes@uv.es 


\section{Introducción}

En el siguiente artículo se presenta una síntesis de los aspectos convergentes que pueden encontrarse entre el pensamiento crítico y las cuestiones sociocientíficas (CSC). En efecto, las reflexiones descritas a continuación son producto del estudio del concepto de pensamiento crítico, desde la filosofía crítica y la didáctica de las ciencias; por ejemplo, desde la filosófica se manifiesta que la principal característica del pensamiento crítico es la duda, la sospecha y el escepticismo aplicado a todo y, en particular, a los discursos y las acciones que se reproducen y se legitiman como verdaderas. Este discurso contribuye a cuestionar la concepción cientificista de la ciencia que se muestra como neutral, lineal e incuestionable (Habermas, 1972; Marcuse, 1964).

La didáctica de las ciencias, a partir de diferentes estudios (Vieira y Nascimento, 2007; Jimenez-Aleixandre, 2010; Vílchez, 2009; Solbes y Vilches, 2004; Kolsto, 2006), toma elementos mediante los cuales podemos cuestionarnos la necesidad de implicarnos en discusiones públicas sobre asuntos importantes que se relacionan con la ciencia y la tecnología. Estos autores coinciden al afirmar que cuando se trabaja sobre una cuestión problemática es posible mostrar el papel social de la ciencia, tanto en sus aspectos internos como externos; lo cual permite el desarrollo del pensamiento crítico.

Otros estudios (Aikenhead, 2005; Ratcliffe y Grace, 2003; Cachapuz, Gil, Carvalho, Praia, y Vilches, 2005) señalan la importancia de considerar elementos controversiales que permitan desarrollar habilidades de argumen- tación, tomar decisiones adecuadas y fomentar el trabajo cooperativo; además de utilizar una metodología para desarrollar pensamiento crítico e independencia intelectual. En este sentido, autores como Jiménez-Aleixandre (2010) adelantan investigaciones en el campo de las cuestiones socio-científicas (CSC), como estrategia pedagógica y didáctica para promover procesos argumentativos. En efecto, esta autora señala que el pensamiento crítico "es la capacidad de desarrollar una opinión independiente, adquiriendo la facultad de reflexionar sobre la sociedad y participar en ella" (p. 39); lo que trae consigo la necesidad del uso y la búsqueda de pruebas, que, junto con el cuestionamiento de la autoridad, son parte fundamental de la argumentación y del pensamiento crítico. Por otra parte, Vieira, Tenreiro-Vieira y Martins (2010) señalan que algunas capacidades - por ejemplo la de cuestionar la validez de los argumentos, rechazar conclusiones no basadas en razones válidas, detectar tendencias, errores de pensamiento y evaluar la credibilidad de la fuentes de información-, son contribuciones importantes para el pensamiento crítico.

Estas perspectivas permiten valorar varios elementos sobre el aporte de las CSC al desarrollo del pensamiento crítico, tales como el establecimiento de juicios fundamentados, el cuestionamiento de la información, la emisión de opiniones y la responsabilidad social. En este sentido, para el desarrollo del pensamiento crítico, el abordaje de las CSC constituye una estrategia para posibilitar juicios fundamentados que requieren abordar cuestiones de manera abierta, conocer diferentes posturas, analizarlas, confrontarlas y valorar sus implicaciones (Solbes y Torres, 2013). 
En virtud de lo anterior, Sadler y Zeidler (2005) consideran que las CSC ayudan a los estudiantes a comprender situaciones sociales, los actos humanos y las cuestiones que por ellas son producidas. Lo mismo consideran Albe, (2008), Chin (2007), Tiberghien, Vince y Gaidioz (2009), quienes manifiestan que la elaboración de los conocimientos que se les enseñe a los estudiantes, conduce a la cultura científica y la educación para la ciudadanía, algo que va más allá de los objetivos disciplinarios.

\section{Desarrollo}

El análisis presentado es producto del desarrollo y discusión de una guía introducto- ria sobre pensamiento crítico y cuestiones socio-científicas. Esta guía presenta el concepto de pensamiento crítico desde perspectivas psicológicas, filosóficas y de didáctica de las ciencias. El desarrollo de la guía se realizó en el área de didáctica de las ciencias, en una universidad colombiana y con 56 estudiantes del programa de Licenciatura en Ciencias Naturales y Educación Ambiental (LCNEA), en grupos de trabajo de 3 y 4 estudiantes.

En este contexto, se presentan en la tabla 1 algunos de los cuestionamientos realizados en la actividad:

Tabla 1. Respuestas identificadas en relación con contextualización sobre pensamiento crítico

\begin{tabular}{|c|c|c|c|}
\hline \multicolumn{4}{|c|}{$\begin{array}{l}\text { 1. Con base en la contextualización anterior, escribe con tus palabras ¿Qué consideras que es el pensamiento crítico y } \\
\text { cuales son características? }\end{array}$} \\
\hline $\begin{array}{l}\text { Es el establecimiento de } \\
\text { juicios fundamentados } \\
\text { desde el cuestionamiento de } \\
\text { distintos puntos de vista. }\end{array}$ & $\begin{array}{l}\text { Capacidad de desenvol- } \\
\text { verse de manera justa, } \\
\text { responsable y flexible. }\end{array}$ & $\begin{array}{l}\text { Forma de aprendizaje con } \\
\text { contribuciones a la autono- } \\
\text { mía, la reflexión y la toma de } \\
\text { decisiones. }\end{array}$ & $\begin{array}{l}\text { Otros: } \\
\text { - Forma de interpretar la } \\
\text { realidad, y sometimiento de } \\
\text { ideas propias a estándares } \\
\text { intelectuales }\end{array}$ \\
\hline G1, G3, G6, G10, G18 & G8, G11 & G2, G5, G9, G13, G15, G17 & G4, G7, G14, G16 \\
\hline
\end{tabular}

Observamos que la tabla anterior encierra aspectos como el establecimiento de juicios fundamentados, el cuestionamiento de la información, la emisión de opiniones y la responsabilidad social. Desde esta mirada, podemos señalar que las características aquí destacadas por los estudiantes, en torno a su conceptualización del pensamiento crítico, son semejantes a las que aparecen en distintos trabajos en el ámbito de las CS. A manera de ejemplo, se presenta la intervención que hace el G10.
La respuesta del grupo 10 se describe a continuación:

G10. "El pensamiento crítico es un cuestionamiento de diferentes puntos de vista y un enfoque propio ante situaciones del contexto, convirtiéndose en una necesidad social de cada persona".

Cuando los estudiantes afirman que es una contribución al cuestionamiento de diferentes puntos de vista, esto coincide con investiga- 
ciones Abd-El-Khalick (2003), que indican que las CSC, por considerar aspectos positivos y negativos, requieren la búsqueda contante de la información que prepara a los estudiantes para emitir opiniones fundamentadas. El mismo autor señala que los problemas sociocientíficos son poco delimitados, multidisciplinares y heurísticos; están además cargados de valores que involucran aspectos políticos, ideológicos, estéticos, ecológicos, morales, educacionales, culturales y religiosos; en cuyo caso posibilitan el cuestionamiento de distintos puntos de vista.

De esta forma, el abordaje de las CSC requiere un análisis de distintas posturas que necesariamente involucran diversos aspectos, tales como lo social, lo político, lo ético, etc.; por lo que no se focaliza únicamente en los conocimientos científicos, sino que es una estrategia que permite vincular situaciones con nociones científicas que tienen impacto con lo social.

Del mismo modo, el pensamiento crítico requiere un posicionamiento que surge del cuestionamiento de diversos puntos de vista, lo que permite apreciar la veracidad o la falsedad de la información y considerar una mirada multidimensional que permite comprender una situación desde diversas dimensiones. Esto constituye una oportunidad para valorar diversos saberes y conocimientos que favorecen formas de reconocimiento cultural.

Otra de las afirmaciones hechas por los estudiantes del grupo 13, se refieren a que el pensamiento crítico es una forma de aprendizaje que genera contribuciones a la autonomía. Así, en el ámbito de las CSC, los estudios de Zeidler, Walker, Ackett y Simmons (2002) han demostrado la importancia del uso de las CSC en el aula, tanto para el aprendizaje de los conceptos científicos como para mejorar la capacidad argumentativa de los estudiantes; al validar información importante para el aprendizaje de conceptos científicos y promover espacios de participación activa que pueden tener contribuciones importantes al desarrollo de la autonomía de los estudiantes, como se aprecia en la siguiente afirmación enunciada por el grupo G13.

G13. Es la capacidad que tiene una persona para indagar, tomar una postura y reflexionar sobre un determinado tema. Con esto se alcanza la autonomía y el desarrollo de las habilidades necesarias para tomar decisiones en un contexto determinado. Se pueden entonces solucionar problemas para llegar a ser personas activas en el ámbito social, político y científico.

Otras investigaciones (Lederman, 2006; Acevedo, 2007) señalan la necesidad que tienen las instituciones educativas de promover cambios de actitud y comportamiento en los estudiantes, de forma que les permitan asumir posiciones sólidas respecto a problemáticas ambientales y sociales, y hacer frente a los problemas socioambientales que afectan a la humanidad.

En este mismo sentido, el estudio de Martínez (2010) muestra que los profesores de ciencias, al trabajar con CSC en sus clases, reflexionan sobre su autonomía profesional y cuestionan la ideología tecnicista del currículo tradicional. Igualmente, Rudduck (1986) indica que los alumnos deben ser ayudados a enfrentar una controversia, a formular opiniones y a tomar decisiones, e impedir que 
cualquier autoridad pueda decidir y resolver en su lugar. Por su parte, Ratcliffe y Grace (2003) señalan que las CSC involucran la formación de opiniones y la realización de decisiones a nivel personal y social.

Otra de las afirmaciones, efectuadas por el G16, señala que el pensamiento crítico es una contribución para apreciar la realidad sin prejuicios:

G16. El pensamiento crítico es una forma de interpretar la realidad, alejada de los prejuicios o creencias. Nos permite explorar nuestro entorno de manera diferente, conocer más allá de lo que comúnmente vemos.

En la interpretación anterior se evidencia que la formulación de estos juicios limita la realidad de la situación, debido a que no se consideran evidencias ni elementos que apoyen una mirada integral acerca de una situación. En este sentido, Yager (1993) relaciona el pensamiento crítico con la capacidad de hacer elecciones racionales y juicios fundamentados como elementos de las decisiones que se emplean para resolver problemas. El pensamiento crítico, al igual que las CSC, se basa en ir más allá de las impresiones y opiniones particulares, lo cual implica cambiar la mirada de aquel que acepta todo lo que se le dice, por la de quien busca el porqué de las cosas.

\section{Algunos aspectos convergentes entre el pensamiento crítico y las cuestiones sociocientíficas}

En la siguiente figura se exponen los aspectos mencionados, a partir de la perspectiva del pensamiento crítico y su relación con las CSC, desde las afirmaciones de los grupos, como producto de la conceptualización de este pensamiento crítico por parte de los estudiantes.

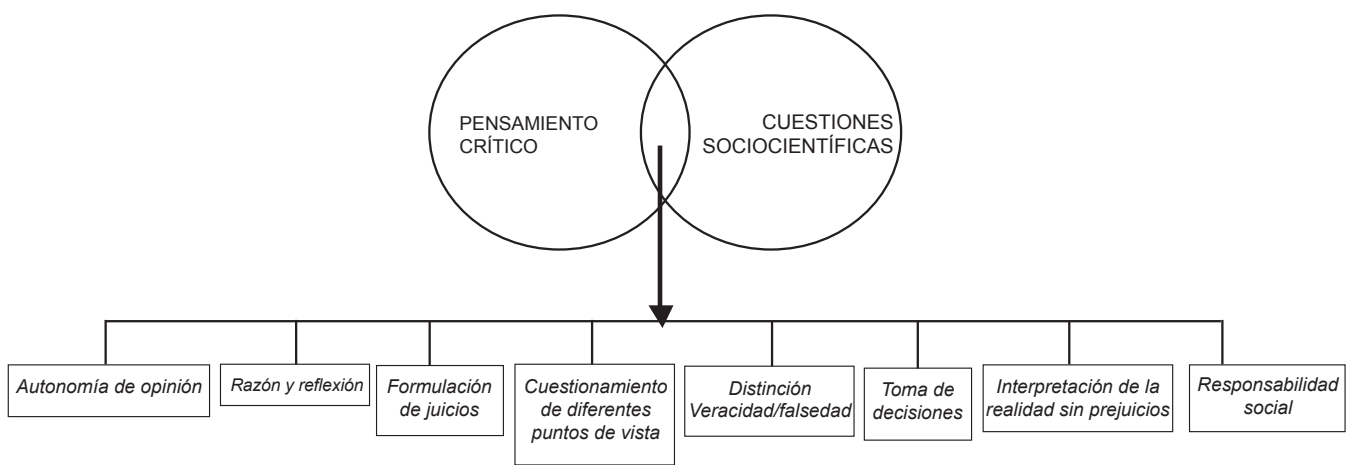

Figura 1. Aspectos convergentes entre el pensamiento crítico y las CSC

Fuente: elaboración propia.

Como se presenta en la figura, tanto las CSC como el pensamiento crítico tienen elementos comunes: promueven la autonomía de opinión, el análisis y la reflexión de situaciones, la for- mulación de juicios fundamentados, el cuestionamiento de la información, la distinción entre lo verdadero y lo falso, la toma de decisiones, la comprensión de la realidad desde la 
integralidad y sin prejuicios, y la actuación de las personas con responsabilidad social.

De igual forma, los participantes señalan que la observación, indagación, interpretación, resolución de problemas, toma de decisiones y cuestionamiento forman parte de las características que los estudiantes destacan del pensamiento crítico. Estas características se constituyen en elementos importantes para valorar la veracidad de los argumentos. Indican que la investigación así como la distinción y valoración de alternativas son contribuciones importantes para fundamentar argumentos coherentes. De esta forma, el pensamiento crítico es una oportunidad para aprender sobre situaciones desde diferentes aspectos, entender sus condiciones, causas, personas involucradas, limitaciones y alcances. Estas mismas características también son contribuciones de las CSC como lo sugieren distintas investigaciones (Martínez, 2010; Torres y Martínez, 2012; Solbes y Torres, 2013; Zeidler et al., 2002).

Otros, grupos como el grupo G12, indican que la coherencia es un componente importante en el pensamiento crítico.

G12: "Para llegar a construir este pensamiento debemos identificar y elaborar explicaciones convincentes dados a un lenguaje apropiado que tengan coherencia con una justificación relevante frente a la toma de decisiones y a la manera de actuar".

El G3 señala que el pensamiento crítico permite dudar de la publicidad. Hacen énfasis en cómo somos manipulados y estamos sometidos a intereses económicos de los medios de comunicación. Desde esta mirada, cobra importancia el cuestionamiento de información así como la formulación de argumentos respaldados por datos que promuevan espacios de crítica y refutación de información.

G3: El pensamiento crítico es el que permite dudar de lo que dicen los medios de comunicación, que hacen que se creen necesidades para que compren productos, en muchos de los casos no necesitados, pero que con la publicidad se crean estas necesidades o solo por el hecho de estar como los demás. Esto solo contribuye a tomar malas decisiones.

\section{Consideraciones finales}

Este análisis reconoce que las CSC favorecen una mirada multidimensional que abarca aspectos de orden social, político, económico y científico; al igual que el pensamiento crítico, el cual permite poner en tela de juicio nuestras ideas, considerando que se realiza una inmersión de la cuestión para comprenderla en cada contexto.

Es de anotar que, dentro de los conceptos manejados en el instrumento de contextualización, el que más llamo la atención por parte de los estudiantes fue el de Ennis (1987), el cual establece que el pensamiento crítico involucra dos características: la razón y la reflexión. Este autor manifiesta que el pensamiento crítico es de orden superior, y, como tal, no es automático sino que requiere de elementos como la autodeterminación y reflexión, esfuerzo, autocontrol y metacognición, puesto que en su ejecución se evalúa no solo el resultado del pensamiento, sino 
también el proceso mismo del pensamiento, elemento necesario también en las CSC.

Es claro que el pensamiento crítico se forja a medida que se posibilitan espacios de práctica en el aula que permitan su desarrollo, en aspectos como la construcción de juicios fundamentados sobre un tema más específico, el cuestionamiento permanente de las cosas, la participación activa en debates, las valoraciones éticas, la formulación de conclusiones adecuadamente sustentadas y por las características descritas de las CSC, que se constituyen en una herramienta didáctica de contribución importante al desarrollo del pensamiento crítico.

\section{Referencias}

Abd-el-Khalick, F. (2003). Socioscientific issues in pre-college science classrooms: the primacy of learners' epistemological orientations and views of nature of science. En Zeidler, D. (Org.). The role of moral reasoning on socioscientific issues and discourse in science education (pp. 41-61). The Netherlands: Kluwer Academic Publishers.

Acevedo, J. (2007). Las actitudes relacionadas con la ciencia y la tecnología en el estudio PISA 2006. Revista Eureka sobre Enseñanza y Divulgación de lasCiencias, 4(3), 394-416.

Albe, V. (2008). When Scientific Knowledge, Daily Life Experience, Epistemological and Social Considerations Intersect: Students' Argumentation in Group Discussions on a Socio-scientific Issue. Research in Science Education, 38, 67-90.
Aikenhead, G. (2005). Educación CienciaTecnología-Sociedad (CTS): una buena idea o como quiera que se le llame. Educación Química, 16(2), 114-124.

Cachapuz, A.; Gil, D.; Carvalho, A.; Praia, J. y Vilches, A. (Coord.). (2005). A necessária renovação do Ensino de Ciências. São Paulo: Cortez.

Chin, C. (2007). Teacher questioning in science classrooms: Approaches that stimulate productive thinking. Journal of Research in Science Teaching, 44(6), 815-843.

Ennis, R. (1985). A logical basis for measuring critical thinking skills. En Educational Leadership, 43(2), 44-46.

Jiménez-Aleixandre, M. (2010). 10 ideas clave. Competencias en argumentación y uso de pruebas. Barcelona: Graó.

Habermas, J. (1972). Teoría analítica de la ciencia y dialéctica. En Adorno, T.; Popper, K.; Dahrendorf, R.; Habermas, J.; Albert, H.; Pilot, H. La disputa del positivismo en la sociología Alemana. Barcelona: Grijalbo.

Kolstø, S. D. (2006). Patterns in students' argumentation confronted with a risk-focused socio-scientific issue. International Journal of Science Education, 28 (14), 1689-1716.

Lederman, N. (2006). Research on nature of science: reflections on the past, anticipations of the future. En AsiaPacific Forum on Science Learning and Teaching, 7(1). Recuperado de http:// www.ied.edu.hk/apfslt/. 
Marcuse, H. (1994). El hombre unidimensional. Barcelona: Ariel.

Martinez, L. (2010). A abordagem de questões sociocientíficas na formação continuada de professores de ciências: contribuições e dificuldades. Tesis Doctoral. Universidade Estadual Paulista. Campus Universitário de Bauru.

Ratcliffe, M. y Grace, M. (2003). Science education for citizenship: teaching socioscientific issues. Philadelphia: Open University Press.

Rudduck, J. (1986). A strategy for handling controversial issues in the secondary school. En Wellinton J. Controversial issues in the curriculum (pp. 6-18). Oxford: Basil Blacckwell.

Sadler, T. D. y Zeidler, D. L. (2005). Patterns of informal reasoning in the context of socioscientific decision making. En Journal of Research in Science Teaching, 42(1), 112-138.

Solbes, J y Torres, N. (2013): ¿Cuáles son las concepciones de los docentes de ciencias en formación y en ejercicio sobre el pensamiento crítico? Revista Tecné, Episteme y Didaxis, 33, 61-85.

Solbes, J. y Vilches, A. (2004). Papel de las relaciones entre ciencia, tecnología, sociedad y ambiente en la formación ciudadana. Enseñanza de las Ciencias, 22(3), 337-348.
Tiberghien, A., Vince, J., E Gaidioz, P. (2009). Design-based Research: case of a teaching sequence on mechanics. International Journal of Science Education, 31(17), 2275-2314.

Torres, N. y Martinez, L. (2011). Desarrollo del pensamiento crítico en estudiantes de Fisioterapia, a partir del estudio de las implicaciones socio-científicas de los xenobioticos. Tecne, Episteme y Didaxis, $29,65-84$.

Vieira, R. y Nascimento, S. (2007). A argumentação no discurso de um professor e seus estudantes sobre um tópico de mecânica newtoniana. En Caderno Brasileiro de Ensino de Física, 24(2), 174-193.

Vílchez, J. E. (2009). La problemática ambiental en los medios. Propuesta de un protocolo de análisis para su uso como recurso didáctico. Enseñanza de las ciencias, 27(3), 421-432.

Yager, R. E. (1993). Science and critical thinking. En Clarke, J. H. y Biddle, A.W. (Eds.), Teaching critical thinking: Reports from across the curriculum. Englewood Cliffs, NJ: Prentice Hall.

Zeidler, D.L.; Walker, K.A.; Ackett, W. A., y Simmons, M. L. (2002). Tangled up in views: Beliefs in the nature of science and responses to socioscientific dilemmas. Science Education, 86(3), 343-367. 